\title{
A Theoretical-Experimental Comparison of CdSe Quantum Dot Optical Properties
}

\author{
Ignacio Gonzalez Oliva, Sandra Alvarenga, Carlos Rudamas \\ Laboratorio de Espectroscopia Optica, Escuela de Fisica \\ Facultad de Ciencias Naturales y Matem'aticas, Universidad de El Salvador \\ Final 25 Av. Norte, San Salvador, El Salvador, Central America \\ go10002@ues.edu.sv; ar12015@ues.edu.sv; carlos.rudamas@ues.edu.sv
}

\begin{abstract}
In this work, we have studied the optical properties of CdSe quantum dots (QDs) with different sizes. Using the SIESTA code and the Kramers-Kronig relations, we have computed the imaginary part of the dielectric constant and the density of states (DOS). The absorption spectra are compared to experimental results from samples fabricated using the thermal decomposition method and a good agreement was obtained. The experimental band edge absorption could be associated to a specific optical transition in our QDs. A well defined second absorption band has been observed in our theoretical results. The energy maximum of these bands follow the expected quantum size effect. However, we do not observed the increase of the energy difference between them, reported by other authors. The reducing of absorption band intensity when the quantum dot size increases, has been seen. Preliminary density of states calculations, also reported in this work, allowed the association of Cd- or Se-character to the energy states in our samples.
\end{abstract}

Keywords: DFT, SIESTA, quantum dots, optical properties, CdSe

\section{Introduction}

The study of CdSe quantum dots (QDs) is one of the main topics of research in solid state physics and material science due to interesting electronic and optical properties that they possess [1]. During the last years, many experimental and theoretical results have been published reporting on intrinsic and extrinsic electronic and optical properties and their dependence on QD size, shape, method of preparation, among others [1, 2, 3, 4, 5, 6]. CdSe QDs are of such great importance because they can be used in so many applications like bioimaging, lasing, diodes, photovoltaics, etc [2]. In previous works, we reported on the characterization of the optical properties and surface defects of colloidal CdSe QDs [6, 7] by using absorption and photoluminescence spectroscopy. Despite of these results, and because of the complexity of systems like colloidal QDs, many optical and electronic properties remain still unknow. In this work, we apply the density functional theory (DFT) in order to have a better understanding of the optical and electronic properties of our samples, by using the SIESTA code [8]. Optical calculations can be performed by using the first order time-dependent perturbation theory to calculate the dipolar transition matrix elements between occupied and unoccupied single-electron eigenstates [9, 10]. With the obtained theoretical results some of the experimental spectral features observed could be explained, even though, DFT could underestimate the value of the HOMO-LUMO when compared with TD-DFT calculations [11].

\section{Calculation and experimental details}

The calculations as stated above were performed using the SIESTA code [8] adding the use of the Kramers-Kronig relations, we have performed calculations of the imaginary part of the dielectric constant. We have used, for the exchange correlation functional, the local density approximation (LDA) within DFT as implemented in the local orbital with the Ceperley-Alder formulation [12]. Pseudopotentials of the Troullier-Martins type [13] and a double-zeta plus polarization (DZP) basis set with an energy shift of $65 \mathrm{meV}$ were used. For the optical calculations, the polycrystal polarization type was selected with an optical mesh of $10 \times 10 \times 10$ and a broaden of $0.1 \mathrm{eV}$. All the simulated samples correspond to plain CdSe nanostructures. An initial bulk of approximately 2000 atoms was reduced by delimiting the xyz coordinates, obtaining a wurtzite sample of 172 atoms, as shown in Fig. 1a. The radius of this sample was estimated to be $1.3 \mathrm{~nm}$ [14, 15]. Once the main sample was designed, an arbitrary reduction of the atoms was performed. Fig. 1b and Fig. 1c show two 
samples with approximately radius of $1.2 \mathrm{~nm}$ and $1.0 \mathrm{~nm}$, respectively. While performing the simulation, molecular dynamics were not implemented but a gradient optimization was executed [14].

The experiments were performed on two solutions containing colloidal quantum dots. They were synthesized by using the thermal decomposition method, similar as Boatman et al [16]. The fabrication has been reported in previous publications $[6,7]$. The radius of the experimental quantum dots was estimated using the semi empirical model of Yu et al. and the modification of Bacherinkov et al. $[17,18]$. The estimated radii of two experimental QDs samples are $1.23 \mathrm{~nm}$ and $1.36 \mathrm{~nm}$.

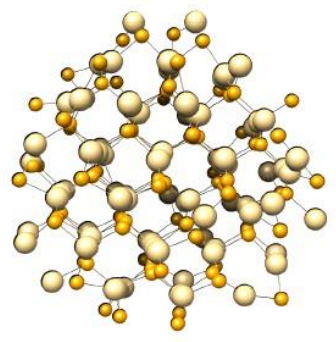

(a)

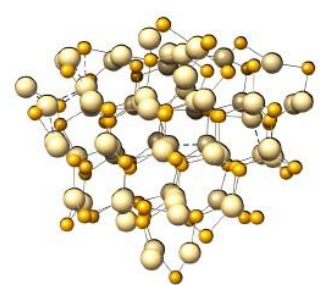

(b)

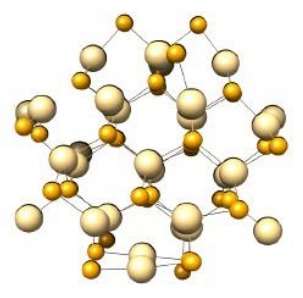

(c)

Fig. 1: (a) The sample $\mathrm{Cd}_{86} \mathrm{Se}_{86}$ has 172 atoms, 86 atoms of $\mathrm{Cd}$ (light yellow) and 86 of Se (yellow) with a radius of $1.3 \mathrm{~nm}$. (b) The sample $\mathrm{Cd}_{62} \mathrm{Se}_{62}$ has 124 atoms, 62 atoms of $\mathrm{Cd}$ and 62 atoms of Se with a radius of $1.2 \mathrm{~nm}$. (c) The sample $\mathrm{Cd}_{34} \mathrm{Se}_{34}$ has 68 atoms, 34 atoms of $\mathrm{Cd}$ and 34 atoms of $\mathrm{Se}$ with a radius of $1.0 \mathrm{~nm}$.

The absorption spectra was measured using a USB4000-UV-VIS spectrograph manufactured by Ocean Optics with a spectral resolution around $1.50 \mathrm{~nm}$, spectral range from $200 \mathrm{~nm}$ to $850 \mathrm{~nm}$ and an entrance slit of $25 \mathrm{~mm}$. As light source we used a tungsten lamp of the same brand.

\section{Results and discussion}

In Fig. 2a, we computed the imaginary part of the dielectric constant. The lower energy absorption peak, inside the visible range, corresponds to $2.39 \mathrm{eV}, 2.29 \mathrm{eV}$ and $2.21 \mathrm{eV}$ for $\mathrm{Cd}_{34} \mathrm{Se}_{34}, \mathrm{Cd}_{62} \mathrm{Se}_{62}$ and $\mathrm{Cd}_{86} \mathrm{Se}_{86}$, respectively.

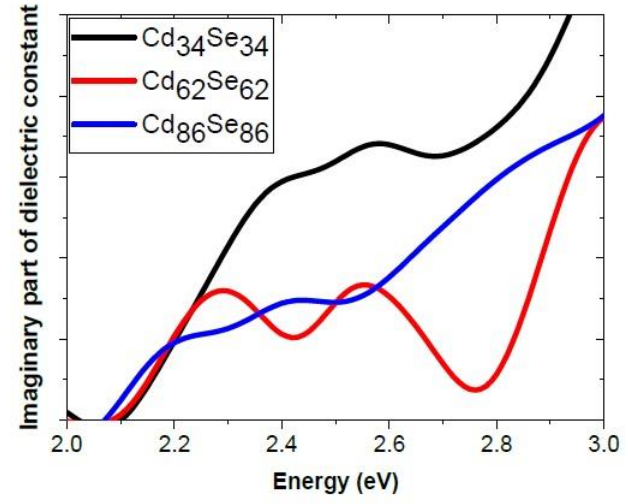

(a)

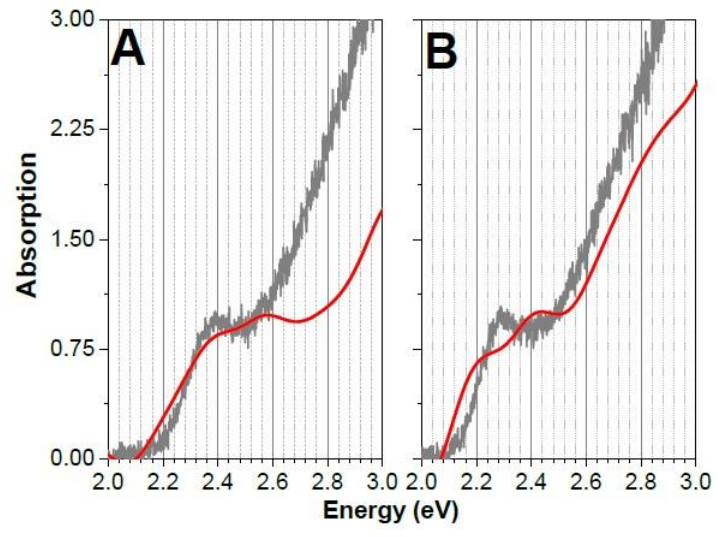

(b)

Fig. 2: (a) Imaginary part of the dielectric constant for $(\mathrm{CdSe})_{n}$ where $n=34 ; 62 ; 86$. (b) A theoretical and experimenta comparison of the lower energy absorption peak. A Experimental (gray) and theoretical (red) $\left(\mathrm{Cd}_{34} \mathrm{Se}_{34}\right)$ normalized absorption spectra for the samples with radii of $1.23 \mathrm{~nm}$ and $1.0 \mathrm{~nm}$, respectively. B Experimental (gray) and theoretical (red) $\left(\mathrm{Cd}_{86} \mathrm{Se}_{86}\right)$ normalized absorption spectra for the samples with radii of $1.36 \mathrm{~nm}$ and $1.30 \mathrm{~nm}$, respectively.

These results are in concordance with the expected quantum confinement effect, when the size of the samples is reduced then the energy of the first absorption peak is increased. Our samples show a clearly behavior of spatial confinement. Similar results were reported by other authors [19]. Their simulations also showed that when the radius of the quantum dots is increased, the intensity of the imaginary part of the dielectric constant is reduced, as we can also observe 
in our results. This could be explained by the increase of the oscillator strength when the confinement increases. The lower absorption peaks for our experimental samples, Fig. $2 \mathrm{~b}$, are $2.43 \mathrm{eV}$ and $2.33 \mathrm{eV}$ that corresponds to the sizes of $1.23 \mathrm{~nm}$ and $1.36 \mathrm{~nm}$, respectively. We can notice that the form of the experimental and theoretical absorption spectra are in very good agreement. One of the main difference between the theoretical and experimental results is the appearance of a second absorption peak at higher energies in the theoretical outcome. The energies of these second absorption peaks are $2.58 \mathrm{eV}$, $2.55 \mathrm{eV}$ and $2.43 \mathrm{eV}$ for $\mathrm{Cd} 34 \mathrm{Se} 34, \mathrm{Cd} 62 \mathrm{Se} 62$, and $\mathrm{Cd} 86 \mathrm{Se} 86$ respectively. The quantum confinement effect for this second peak can also be observed. The lower and higher energy peaks obtained could be associated to $1 \mathrm{~S}_{3 / 2}-1 \mathrm{~S}_{\mathrm{e}}$ and $2 \mathrm{~S} 3 / 2-1 \mathrm{~S}$ electron-hole's transition after the results obtained by other authors [21]. Despite the fact that our experimental samples do not show this second absorption peak, other publications have reported it, for CdSe nanocrystals [20, 21]. According to Ekimov et al. [21], the difference in energy, between the lower and higher energy absorption peaks, increases when the size is reduced. We cannot observe this effect clearly since the difference in our results are $0.229 \mathrm{eV}$ for $\mathrm{Cd} 86 \mathrm{Se} 86,0.264 \mathrm{eV}$ for $\mathrm{Cd} 62 \mathrm{Se} 62$ and $0.184 \mathrm{eV}$ for $\mathrm{Cd} 34 \mathrm{Se} 34$. The discrepancies between the theoretical and experimental results could be related to the following situations: (i) no molecular dynamics have been performed, (ii) ligands in colloidal quantum dots, which are not taken into account in the calculations, play an important role in the electronic states and (iii) temperature related effects.

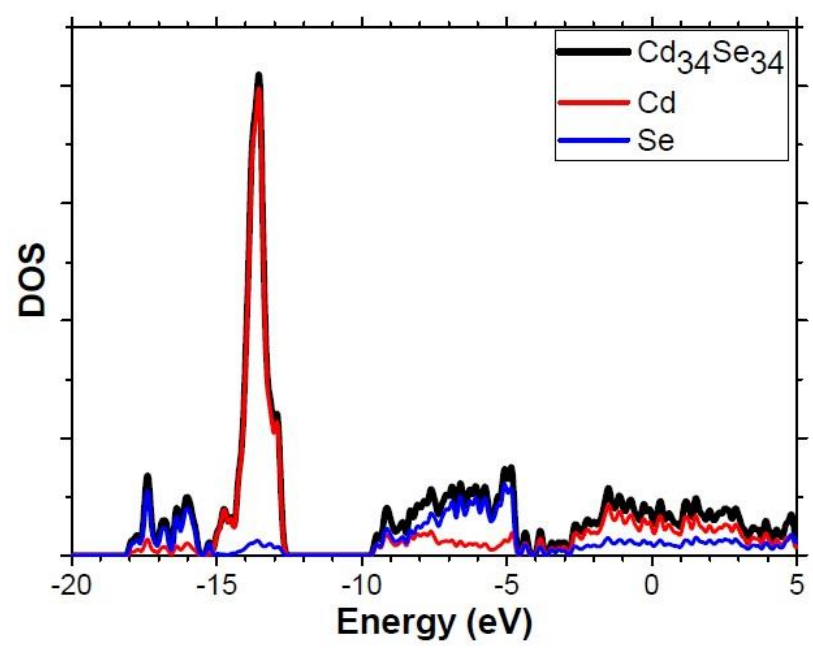

Fig. 3: The density of states for the $\mathrm{Cd}_{34} \mathrm{Se}_{34}$ structure.

The use of the density of states (DOS) can also explain the transitions between the discrete levels in the quantum dot [22]. In Fig. 3, we present preliminary results of the density of states for the Cd34Se34 sample. The density of states are in good agreement with previous studies. We obtained a Cd character and a Se character for the states from $-16 \mathrm{eV}$ to $-14 \mathrm{eV}$ and from $-10 \mathrm{eV}$ to $-5 \mathrm{eV}$, respectively. Similar results have been obtained by Deglmann et al and Voznyy et al. [23, 4, 5]. On the other hand, a Cd-character can be associated to the energy states located from $-3 \mathrm{eV}$ to $3 \mathrm{eV}$.

\section{Conclusions}

In this work, we conclude that the use of the density functional theory as implemented by SIESTA is an acceptable tool for calculating the imaginary part of the dielectric constant of CdSe nanostructures. The quantum confinement effect can be easily studied under the procedure described in this research. The comparison between theoretical and experimental results has been used to understand some of the band edge properties of our samples. The calculation of imaginary part of the dielectric constant presents two well defined peaks that behaves as in a spatial confinement situation and could be associated to two specific optical transition in our QDs. The density of states in the energies values from $-16 \mathrm{eV}$ to $-14 \mathrm{eV}$ and from $-3 \mathrm{eV}$ to $3 \mathrm{eV}$ is dominated by $\mathrm{Cd}$ character. On the other hand, from $-10 \mathrm{eV}$ to $-5 \mathrm{eV}$, the DOS is dominated by a Se character. 


\section{Acknowledgments}

The authors would like to thank the members of the research group that contributed to the discussion of this paper. This work was partially supported by "Consejo de Investigaciones Cientificas" of the Universidad de El Salvador under the 09.20 project and "Programa Jovenes Talentos".

\section{References}

[1] M. Yu, G. W. Fernando, R. Li, F. Papadimitrakopoulos, N. Shi and R. Ramprasad, "First principles study of CdSe quantum dots: Stability, surface unsaturations, and experimental validation," Appl. Phys. Lett., vol. 88, no. 23, pp. 88-90, 2006.

[2] J. Y. Kim, O. Voznyy, D. Zhitomirsky and E. H. Sargent, "25th Anniversary Article: Colloidal Quantum Dot Materials and Devices: A Quarter-Century of Advances," Adv. Mater., vol. 25, no. 36, pp. 4986-5010, 2013.

[3] S. Rosenthal, J. Macbride, S. Pennycook and L. Feldman, "Synthesis, surface studies, composition and structural characterization of CdSe, core/shell and biologically active nanocrystals," Surf. Sci. Rep., vol. 62, no. 4, pp. 111-157, 2007.

[4] O. Voznyy, "Mobile surface traps in CdSe nanocrystals with carboxylic acid ligands," J. Phys. Chem. C, vol. 115, no. 32, pp. 15927-15932, 2011.

[5] O. Voznyy, S. M. Thon, A. H. Ip, and E. H. Sargent, "Dynamic trap formation and elimination in colloidal quantum dots," J. Phys. Chem. Lett., vol. 4, no. 6, pp. 987-992, 2013.

[6] H. Ponce and C. Rudamas, "Optical characterization of surface defects in cadmium selenide (CdSe) colloidal quantum dots," Revista Materia, vol. 20, no. 3, pp. 676-681, 2015.

[7] M. Pacheco and C. Rudamas, "Fabrication and optical characterization of CdSe quantum dots," in Proceddings of the 62nd Annual Meeting of the Austrian Physical Society, Graz, Austria, p. 159, 2012.

[8] J. M. Soler et al., "The SIESTA method for ab initio order-N materials simulation," J. physics. Condens. Matter, vol. 2745, no. Xc, p. 22, 2002.

[9] A. Segura et al., "Specific features of the electronic structure of III-VI layered semiconductors: Recent results on structural and optical measurements under pressure and electronic structure calculations," Phys. Status Solidi Basic Res., vol. 235, no. 2, pp. 267-276, 2003.

[10] D. S. Portal, P. Ordejon, and E. Canadell, "Computing the properties of materials from first principles with SIESTA," Princ. Appl. Density Inorg. Chem. li, vol. 113, pp. 103-170, 2004.

[11] O. V. Prezhdo, "Photoinduced Dynamics in Semiconductor Quantum Dots: Insights from Time-Domain ab initio Studies," Acc. Chem. Res., vol. 42, no. 12, pp. 2005-2016, 2009.

[12] D. M. Ceperley and B. J. Alder, "Ground state of the electron gas by a stochastic method," Phys. Rev. Lett., vol. 45, no. 7, pp. 566-569, 1980.

[13] N. Troullier and J. L. Martins, "Efficient Pseudopotentials for plane-wave calculations," Phys. Rev. B, vol. 43, no. 3, pp. 1993-2006, 1991.

[14] MarvinSketch, version 6.2.2 , calculation module developed by ChemAxon, 2014, [Online]. Available: http://www.chemaxon.com/products/marvin/marvinsketch/.

[15] E. F. Pettersen et al, "UCSF Chimera - A visualtization system for exploratory research and analysis," J. Comput. Chem., vol. 25, no.13, pp. 1605-1612, 2004.

[16] K. J. Nordell, E. M. Boatman, and G. C. Lisensky, "A Safer, Easier, Faster Synthesis for CdSe Quantum Dot Nanocrystals," J. Chem. Educ., vol. 82, no. 11, p. 1697, 2005.

[17] W. W. Yu, L. Qu, W. Guo, and X. Peng, "Experimental determination of the extinction coefficient of CdTe, CdSe, and CdSe nanocrystals," Chem. Mater., vol. 15, no. 14, pp. 2854-2860, 2003.

[18] Y. Y. Bacherikov, M. O. Davydenko, A. M. Dmytruk, I. M. Dmitruk, and P. M. Lytvyn, "CdSe nanoparticles grown with different chelates," Semicond. Physics, Quantum Electron. Optoelectron., vol. 9, pp. 75-79, 2006.

[19] L.-W. Wang and A. Zunger, "Pseudopotential calculations of nanoscale CdSe quantum dots," Phys. Rev. B, vol. 53, no. 15 , pp. 9579-9582, 1996.

[20] R. Jose, N. U. Zhanpeisov, H. Fukumura, Y. Baba and M. Ishikawa, "Structure-property correlation of CdSe clusters using experimental results and first principles DFT calculations," J. Am. Chem. Soc., vol. 128, no. 2, pp. 629-36, 2006. 
[21] A. I. Ekimov, et al., "Absorption and intensity-dependent photoluminescence measurements on CdSe quantum dots: assingment of the first electronic transitions," J. Opt. Soc. Am. B, vol. 10, no. 1, pp. 100-107, 1993.

[22] L. Chen, H. Bao, T. Tan, O. V. Prezhdo and X. Ruan, "Shape and temperature dependence of hot carrier relaxation dynamics in spherical and elongated CdSe quantum dots," J. Phys. Chem. C, vol. 115, pp. 11400-11406, 2011.

[23] P. Deglmann, R. Ahlrichs, and K. Tsereteli, "Theoretical studies of ligand-free cadmium selenide and related semiconductor cluster," J. Chem. Phys., vol. 116, no. 4, pp. 1585-1597, 2002. 